Method We completed searches of MEDLINE and PSYCInfo from inception of each database to June 2013, to identify primary studies evaluating symptom exaggeration among patients with mental health disorders. Teams of reviewers completed title and abstract screening of citations, full text review of potentially eligible articles, data extraction, and quality assessment of eligible studies. Findings from our review and input from content experts informed the development of a summary instrument.

Results We identified 8435 unique citations; 102 studies were eligible. Studies reported on 55 different instruments to assess symptom exaggeration; $42 \%$ of studies used the MMPI-2. Quality of studies ranged from low to moderate. Items that comprise our summary instrument will be presented at EPICOH.

Conclusions Multiple instruments are available to assess symptom exaggeration among patients presenting with mental illness; however, no instrument has shown ideal psychometric properties. We are hopeful that our instrument will facilitate the testing and development of a novel tool with superior sensitivity and specificity for detecting symptom exaggeration.

\section{DEVELOPMENT OF AN INSTRUMENT ASSESSING RECOVERY EXPECTATIONS IN PATIENTS RECEIVING DISABILITY BENEFITS SECONDARY TO MENTAL HEALTH DISORDERS}

1,2Shanil Ebrahim, ${ }^{3}$ Cindy Malachowski, ${ }^{1}$ Mostafa Kamal el Din, ${ }^{1}$ Sohail Mulla, ${ }^{3}$ Sheena Bance, ${ }^{4}$ Luis Montoya, ${ }^{1}$ Gordon Guyatt, ${ }^{1} J a s o n$ Busse. ${ }^{1}$ McMaster University, Hamilton, Canada; ${ }^{2}$ Stanford University, Stanford, USA; ${ }^{3}$ University of Toronto, Toronto, Canada; ${ }^{4}$ University Health Network, Toronto, Canada

\subsection{6/oemed-2014-102362.252}

Objectives Of workers approved for long-term disability benefits, $31.6 \%$ suffer from a primary mental illness. Negative patient recovery expectations are associated with worse outcome in many conditions. Our objectives were: 1) to complete a systematic review to identify measures that assess patient recovery expectations, and 2) using the results from our review, develop an instrument designed to assess recovery expectations in individuals receiving disability benefits secondary to a mental health disorder.

Method We completed searches of MEDLINE and PSYCInfo from inception of each database to June 2013, to identify primary studies evaluating patient's recovery expectations across all clinical populations. Teams of reviewers completed title and abstract screening of citations, full text review of potentially eligible articles, data extraction, and quality assessment of eligible studies. Findings from the review and input from content experts informed the development of a comprehensive list of items that captured patient's recovery expectations.

Results We identified 12599 unique citations; 46 studies were eligible. Studies reported on 20 different instruments assessing patient's recovery expectations. A minority (20\%) of studies included measurement properties of reported instruments; 11\% evaluated mental health conditions. No instruments were validated among patients receiving disability benefits or explored associations with return to work. Generalizability to disabled patients receiving wage replacement benefits is therefore unclear. Items that comprise our summary instrument will be presented at $\mathrm{EPICOH}$.

Conclusions Our instrument holds promise for identifying claimants holding negative recovery expectations, which may be associated with prolonged recovery. These unhelpful beliefs may be a useful target for early interventional therapies.

\section{SHIFT WORK AND HYPERTENSION: PREVALENCE AND ANALYSIS OF DISEASE PATHWAYS IN GERMAN CAR MANUFACTURING WORKERS}

${ }^{1}$ Johan Ohlander, ${ }^{2}$ Mekail-Cem Keskin, ${ }^{2}$ Joachim Stork, ${ }^{1}$ Katja Radon. ${ }^{1}$ University Hospital Munich, Munich, Bavaria, Germany; ${ }^{2}$ AUDI AG, Ingolstadt, Bavaria, Germany

\subsection{6/oemed-2014-102362.253}

Objectives Triggered by disturbed circadian rhythm, increased blood pressure comprises an intermediate step on suggested pathways between shift work and cardiovascular disease. Despite accumulated evidence indicating an excess risk for cardiovascular disease among shift workers, studies on shift work and hypertension are inconclusive. Thus, we investigated the association between shift work and hypertension in German car manufacturing workers.

Method Baseline blood pressure and potential confounders for 25343 workers aged 16 to 64 years were obtained from standardised medical check-ups. Workers were analysed according to four exposure categories: no shift work, shift work without night shifts, shift work with night shifts and constant night shifts. Cross-sectional associations between shift work and hypertension were analysed using logistic regression adjusted for confounders and sets of behavioural, psychosocial and physiological factors.

Results Hypertension prevalence was highest among shift workers not working night shifts (11.5\%), and lowest among workers not working shifts $(7.8 \%)$. Unadjusted, a significant positive association with hypertension was found for shift work without night shifts (OR 1.53 95\% CI 1.40-1.68) and constant night shifts (OR 1.46, 95\% CI 1.29-1.65). Adjusted for confounders, only shift work without night shifts showed an increased hypertension risk (OR 1.15, 95\% CI 1.02-1.30). However, stratification for occupational status suggested this excess risk to be attributable only to white-collar shift workers not working night shifts (OR 1.52 95\% CI 1.22-1.88). After adjustments for behavioural factors no shift work category showed increased hypertension risks.

Conclusions Shift workers' increased hypertension risk might be explained by behavioural risk factors, possibly triggered by disturbed circadian rhythm.

\section{PRELIMINARY RESULTS OF SHIFT WORK AND CARDIOVASCULAR RISK FACTORS: ANALYSING BASELINE DATA OF A PROSPECTIVE NIGHT SHIFT WORKER COHORT IN SHENZHEN, CHINA}

\footnotetext{
1,2 Shelly Lap Ah Tse, ${ }^{1,2}$ Feng Wang, ${ }^{3}$ Liuzhuo Zhang, ${ }^{3}$ Yanfang Zhang, ${ }^{1}$ Bo Zhang, ${ }^{1,4}{ }^{4}$ Yonghua He, 'Shaohua Xie, ${ }^{1}$ Mengjie Li, ${ }^{3}$ Zhimin Li, 'Ignatius Tak-sun Yu. ${ }^{1} \mathrm{JC}$ School of Public Health and Primary Care, The Chinese University of Hong Kong, Sha Tin, Hong Kong; ${ }^{2}$ Shenzhen Municipal Key Laboratory for Health Risk Analysis, Shenzhen Research Institute of the Chinese University of Hong Kong, Shenzhen, China; ${ }^{3}$ Shenzhen Prevention and Treatment Center for Occupational Diseases, Shenzhen, China; ${ }^{4}$ CUHK Centre for Public Health and Primary Care (Shenzhen), Shenzhen Research Institute of the Chinese University of Hong Kong, Shenzhen, China
}

\subsection{6/oemed-2014-102362.254}

Objectives The incidences of cardiovascular diseases (CVD), diabetes mellitus and metabolic syndrome increase rapidly in China, while the prevalence of night shift work is also increasing. This study aims to investigate the relationship between night shift work and CVD risk factors by analysing the baseline data of a prospective cohort study. 
Method We are establishing a prospective night shift worker cohort in Shenzhen, China. More than 10000 workers will be recruited and followed up. Currently, we have recruited more than 4000 workers but most questionnaires have not yet been input. A standardised questionnaire is used to collect information on lifetime night shift work, light at night, occupational hazards, sleep disorders, etc. Fasting blood and spot urine samples are also collected for further usage. CVD risk factors include hypertension, diabetes, overweight and dyslipidemia etc. All participants will have the annual or biennial occupational physical examination.

Results We reported data from a manufacturing company dealing with welding and shipment. We obtained 131 day workers and 370 shift workers with a response rate of $95 \%$. The shift workers are significantly younger than day workers. The smoking and alcohol drinking status are comparable in two groups. The presence of number of CVD risk factors positively relate to the years of shift work after adjusting for age and other confounding factors.

Conclusions These preliminary results suggest long-term night shift work may increase CVD risk factors, while these will be updated in the conference. [National Natural Science Foundation of China (Project number 81273172 and 81372964), Shelly@cuhk.edu.hk (Lap Ah Tse)]

\section{PRELIMINARY BASELINE CHARACTERISTICS OF A PROSPECTIVE NIGHT SHIFT WORKER COHORT IN SHENZHEN, CHINA}

${ }^{1,2}$ Feng Wang, ${ }^{1,2}$ Shelly Lap Ah Tse, ${ }^{3}$ Liuzhuo Zhang, ${ }^{3}$ Yanfang Zhang, ${ }^{1}$ Bo Zhang,
1,4 ${ }^{4}$ onghua He, ${ }^{1}$ Shaohua Xie, ${ }^{1}$ Mengjie Li, ${ }^{3}$ Zhimin Li, 'Ignatius Tak-sun Yu. ${ }^{1} J C$ School of
Public Health and primary Care, the Chinese University of Hong Kong, Sha Tin, Hong
Kong; ${ }^{2}$ Shenzhen Municipal Key Laboratory for health Risk Analysis, Shenzhen Research
Institute of the Chinese University of Hong Kong, Shenzhen, China; ${ }^{3}$ Shenzhen
Prevention and Treatment Center for Occupational Diseases, Shenzhen, China; ${ }^{4}$ CUHK
Centre for Public health and Primary Care (Shenzhen), Shenzhen Research Institute of the
Chinese University of Hong Kong, Shenzhen, China

10.1136/oemed-2014-102362.255

Objectives The incidence of metabolic syndrome (MS) increases rapidly in China. Growing evidence suggests that alterations in circadian systems and sleep disorders have participated in the etiology of metabolic disorders. This study aims to investigate the relationship between night shift work and MS risk and examine the underlying mechanisms that have never been explored by previous epidemiological studies.

Method We are establishing a prospective night shift worker cohort in Shenzhen, China. More than 10000 participants will be recruited and followed up. A standardized questionnaire is used to collect information on lifetime night shift work, light at night, occupational hazards, sleep disorders, etc. Fasting blood and spot urine samples are also collected for further usage. All participants will have the annual or biennial occupational physical examination. MS will be diagnosed following the NCEP-ATPIII criteria. Currently, we have recruited more than 4000 workers but most questionnaires have not yet been input.

Results We reported data from the occupational health examination. We obtained 3878 male workers and 250 female workers with a response rate of $95 \%$. More than $50 \%$ participants are aged from 20-30 years. About 3.4\% subjects' fasting blood glucose was more than $6.1 \mathrm{mmol} / \mathrm{L}$. More than $30 \%$ subjects are overweight, and $25 \%$ subjects' is with high blood pressure.
Conclusions It is the basic characteristics of the recruited participants. The preliminary result of night shift work and MS will be present in the conference. [National Natural Science Foundation of China (Project number 81273172 and 81372964), Shelly@cuhk.edu.hk (Lap Ah Tse)]

\section{ASSESSMENT OF EXPOSURE TOTETRACHLORETHYLENE AND ITS CLINICAL IMPACT IN A POPULATION OF 60 EMPLOYEESCLEANERS IN WEST ALGERIA}

Zoubida Belhadj, Badreddine Abdelkrim Kandouci, Chahrazed Kandouci, Djelloul Zergoun. UDL Sidi Bel Abbes, Sidi Bel Abbes, Algeria

10.1136/oemed-2014-102362.256

Objectives Evaluation of the clinical impact of chronic exposure to perchlorethylene in west Algeria.

Method After protocol definition, inclusion of salaries for three pressings willayas the west Algeria (Sidi Bel Abbes, Oran and Ain Témouchent), we conducted a study papers / unexposed to evaluate the clinical symptoms with matching on age and sex.

55Data collection made by a questionnaire (one of Hogstedt). Were conducted psychometric tests.

The evaluation of occupational exposure by air sampling was carried out in two pressings using colorimetric detector tubes for spot metering (Dragger pumps). The first in any point in the space, the second opening when the window.

Results Sixty employees and 120 controls were included. 50\% of employees often have fatigue at the end of work against it are more than $50 \%$ sometimes complain of fatigue at the beginning and at the workplace and irritability.

There is a significant difference between exposed and unexposed population on the presence of clinical signs except for the presence of three signs: nightmares, tingling in arms and digestive disorders.

There is a significant difference between the two populations for all the psychometric tests.

Perchlorethylene air rate upon opening of the window is greater than or equal to $100 \mathrm{ppm}$ and both are higher than the limit value ACGIH 2005

Conclusions This study confirms the existence of an occupational exposure to perchlorethylene dry cleaners or employees of the questionnaire results allowed us to identify a number important information on the symptoms observed in the study population according to exposure.

\section{EXPOSURE TO STYRENE AND THE RISK OF CANCER: A LONG-TERM FOLLOW-UP STUDY OF WORKERS IN THE DANISH REINFORCED PLASTICS INDUSTRY}

1,2 MS Christensen, ${ }^{3} \mathrm{~J}$ Hansen, ${ }^{4} \mathrm{CH}$ Ramlau-Hansen, ${ }^{1} \mathrm{G}$ Toft, ${ }^{5} \mathrm{~F}$ d'Amore, ${ }^{1} \mathrm{HA}$ Kolstad. 'Danish Ramazzini Center, Deptartment of Occupational Medicine, Aarhus University Hospital, Aarhus, Denmark; ${ }^{2}$ Centre for Integrated Register-Based Research, CIRRAU, Aarhus University, Aarhus, Denmark; ${ }^{3}$ Danish Cancer Society Research Center, Copenhagen, Denmark; ${ }^{4}$ Department of Public Health, Section for Epidemiology, Aarhus University, Aarhus, Denmark; ${ }^{5}$ Department of Haematology, Aarhus University Hospital, Aarhus, Denmark

\subsection{6/oemed-2014-102362.257}

Objectives Styrene was incorporated in the $12^{\text {th }}$ Report on Carcinogens (RoC) based on sufficient experimental evidence in animals. The human evidence has been evaluated as limited by RoC and IARC. The objective of this study was to analyse the risk of 